Mitteilungen der Österreichischen Geographischen Gesellschaft, 159. Jg. (Jahresband), Wien 2017, S. 368-378

\title{
Der Deutsche Kongress für Geographie 2017 - eine Reflexion der grössten Tagung in der Geschichte TüBINGENS
}

\author{
Peter Alexander Rumpolt, Wien, und Marc Michael SeEbACHER, Frankfurt am Main*
}

mit 7 Abb. im Text

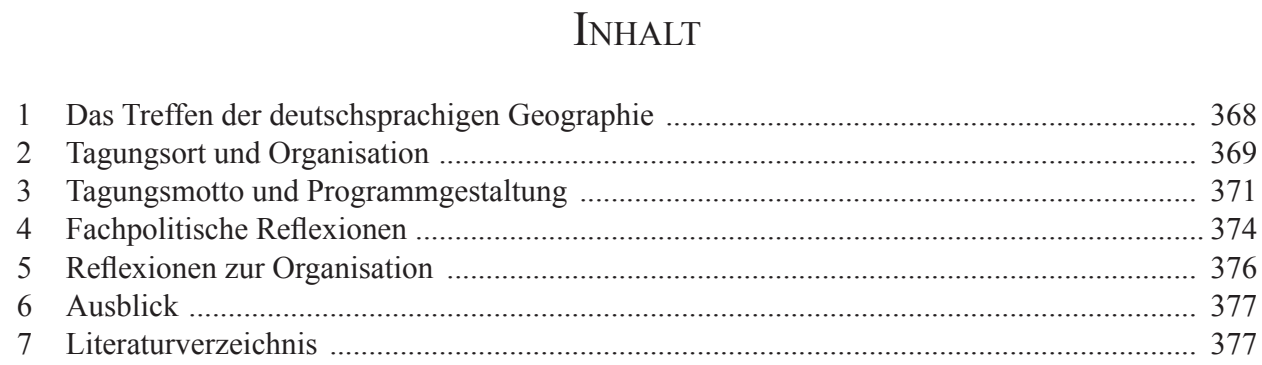

\section{Das Treffen der deutschsprachigen Geographie}

Der bis 2013 als Deutscher Geographentag (DGT) bezeichnete Deutsche Kongress für Geographie (DKG) - die „größte wissenschaftliche Veranstaltung der deutschsprachigen Geographie“ (GAMERITH 2017, S. 11) - wurde im Jahr 2017 zum fünften Mal in Folge als gemeinsame Tagung für Hochschul-, Schul- und Berufsgeographinnen und -geographen abgehalten. Die ersten vier in dieser Form als Großkongress veranstalteten Ausgaben des DGT/DKG hatten 2007 in Bayreuth, 2009 in Wien (siehe Musil 2009), 2013 in Passau (siehe Rumpolt 2013a, 2013b) sowie 2015 in Berlin (siehe Rumpolt \& SeEbacher 2015a, 2015b) stattgefunden. Im Jahr 2017 war nun die schwäbische Universitätsstadt Tübingen Schauplatz dieser für die deutschsprachige Geographie zentralen Tagung; zum zweiten Mal wurde dabei die Bezeichnung Deutscher Kongress für Geographie (DKG) verwendet.

Der DKG 2017 stellt somit sowohl den zweiten Geographentag unter der Bezeichnung Deutscher Kongress für Geographie als auch den fünften, welcher als Großkongress für Wissenschaft, Schule und Praxis durchgeführt wurde, dar. Insgesamt handelt es sich beim Tübinger Kongress um den 60. deutschsprachigen Geographentag (DGT oder DKG), im 21. Jh. um den achten (siehe

\footnotetext{
* Mag. Peter Alexander Rumpolt, Institut für Stadt- und Regionalforschung, Österreichische Akademie der Wissenschaften, Postgasse 7/4/2, A-1010 Wien; E-Mail: peter.rumpolt@oeaw.ac.at, peter.rumpolt@ univie.ac.at, http://www.oeaw.ac.at/isr/; Marc Michael SEeBACHER, BA MA, Institut für Humangeographie, Goethe-Universität Frankfurt am Main, Theodor-W.-Adorno-Platz 6, D-60629 Frankfurt am Main; E-Mail: seebacher@em.uni-frankfurt.de,http://www.humangeographie.de
} 
Rumpolt \& SeEBACher 2017). ${ }^{1)}$ In Bezug auf die Teilnehmerzahl avancierte der DKG 2017 laut Auskunft des Ortsausschuss-Vorsitzenden Sebastian KINDER sogar zur größten Tagung in der Geschichte Tübingens, schlussendlich wurden insgesamt 1.650 Teilnehmer verzeichnet. ${ }^{2)}$

\section{Tagungsort und Organisation}

Den Tagungsort des vom 29. September bis 4. Oktober 20173) durchgeführten Kongresses bildete das in Baden-Württemberg südlich von Stuttgart gelegene Tübingen mit seiner im Jahr 1477 gegründeten Universität. Die zwischen nördlichem Schwarzwald und Schwäbischer Alb reizvoll eingebettete Stadt (Abb. 1) ist Sitz des Landkreises Tübingen und des Regierungsbezirkes Tübingen, des südöstlichen der vier Regierungsbezirke Baden-Württembergs. Das Tagungsgelände im engeren Sinn bildeten das Hauptgebäude der Eberhard-Karls-Universität Tübingen, die Neue Aula (Abb. 2), und weitere zu Fuß überwiegend sehr gut erreichbare Universitätsgebäude im unmittelbaren Umkreis der Neuen Aula.

Tagungsleitung und Vorsitz des Ortsausschusses Tübingen lagen in den Händen von Sebastian Kinder, Koordinatorin des Kongresses war Dana Pietsch. Für die Organisation zeichnete die Conventus Congressmanagement \& Marketing GmbH Jena verantwortlich. Zahlreiche mithelfende Studentinnen und Studenten waren durch orangefarbene T-Shirts mit der Aufschrift DKG-Team gut erkennbar.

Die E-Mail-Aussendungen seitens der Kongressorganisation im Vorfeld des Kongresses erwiesen sich als übersichtlich und sehr professionell. Um im Tagungsbüro rasch einchecken zu können, konnte man einen „Self-check-in-Schalter“ benützen: Ein mit der Buchungsbestätigung erhaltener QR-Code musste unter einen Scanner gehalten werden, wodurch der automatische Ausdruck des Namensschildes ausgelöst wurde. Direkt am Namensschild waren auch alle gebuchten Veranstaltungen wie z.B. Exkursionen angegeben. Exkursionsteilnehmer erhielten den gedruckten Exkursionsführer diesmal ohne Mehrkosten - eine sehr erfreuliche Neuerung! Die schon traditionelle Tagungstasche entpuppte sich als, schlankes' Stoffaccessoire.

1) Bei der Fach- und Verlagsausstellung lag am Stand des Franz-Steiner-Verlages ein erstes Exemplar oder ein Vorabdruck des neuen Geographischen Taschenbuches auf. Die darin enthaltene Information, dass der DKG 2017 als die bereits 61. Ausgabe des Geographentages angesehen werden kann, basiert offensichtlich auf dem Mitzählen des im Jahr 2012 in Köln abgehaltenen International Geographical Congress (IGC; zum 32. Weltkongress der Geographie siehe Nissel \& EmBLEton-Hamann 2012) anstelle der entfallenen Ausrichtung eines Deutschen Geographentages im Jahr 2011. Die Autoren dieses Beitrags vertreten jedoch die Ansicht, den 2012 in Deutschland durchgeführten ,globalen Geographentag' nicht gleichzeitig auch als einen eigenen Deutschen Geographentag zu werten. Demzufolge wäre die Universität Tübingen 2017 Gastgeber des 60 . DGT/DKG, wie dies auch im Programmheft (siehe GAMERITH 2017; KINDER 2017) sowie im Rahmen der Festveranstaltung des DKG 2017 festgehalten wurde.

2) Während in Tübingen im Rahmen der Festveranstaltung eine Teilnehmerzahl von rund 1.800 kolportiert worden war, ergab eine Nachfrage der Autoren bei der Kongressorganisation (Conventus Congressmanagement \& Marketing $\mathrm{GmbH}$ ) eine Teilnehmerzahl von 1.650 (Auskunft per E-Mail am 19.12.2017).

3) Während auf der Webseite http://www.dkg2017-tuebingen.de, am Deckblatt des gedruckten Programmheftes sowie u.a. auch auf den vor dem Hauptgebäude der Eberhard-Karls-Universität Tübingen (Neue Aula; Abb. 2) angebrachten Transparenten der Zeitraum 30.09.-05.10.2017 angegeben war, wurde der Kongress de facto im Zeitraum 29.09.-04.10.2017 abgehalten. Der 29. September war Arbeitskreis- und Verbandssitzungen gewidmet. 


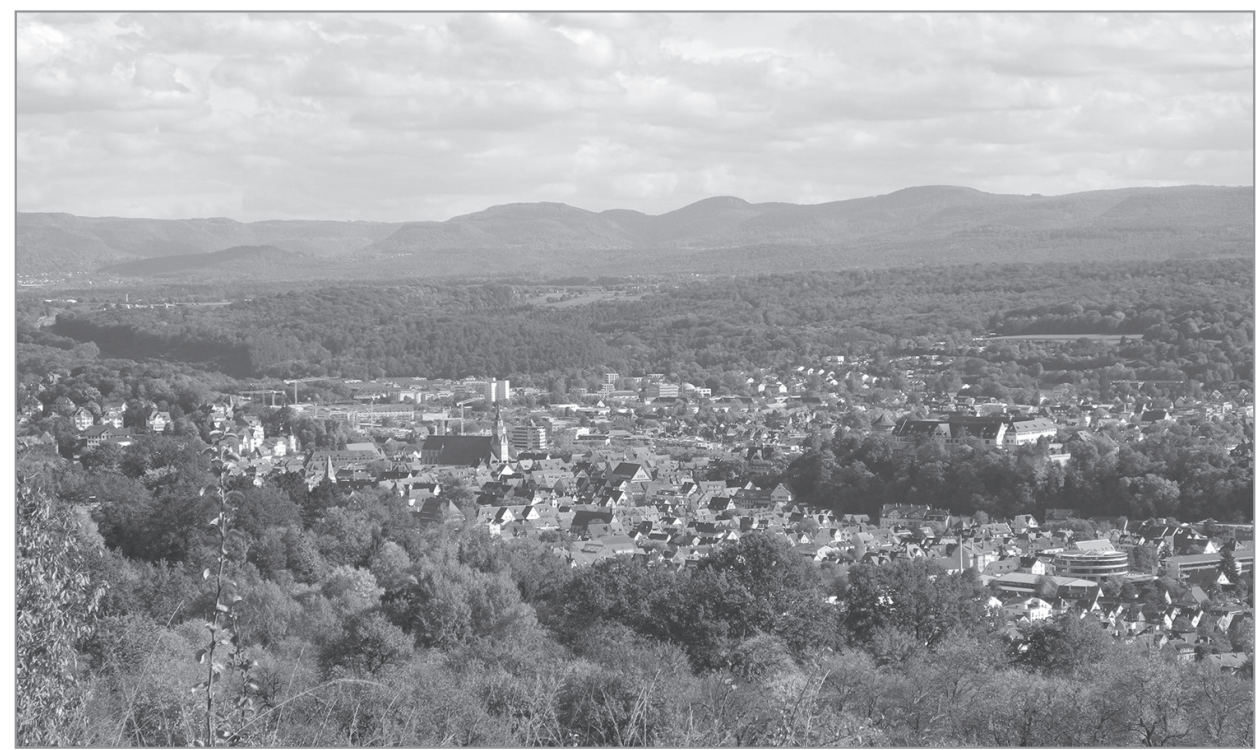

Foto: $\quad$ P.A. Rumpolt 2017

Abb. 1: Blick von Nordwesten auf die Altstadt von Tübingen, im Hintergrund die Schwäbische $\mathrm{Alb}$

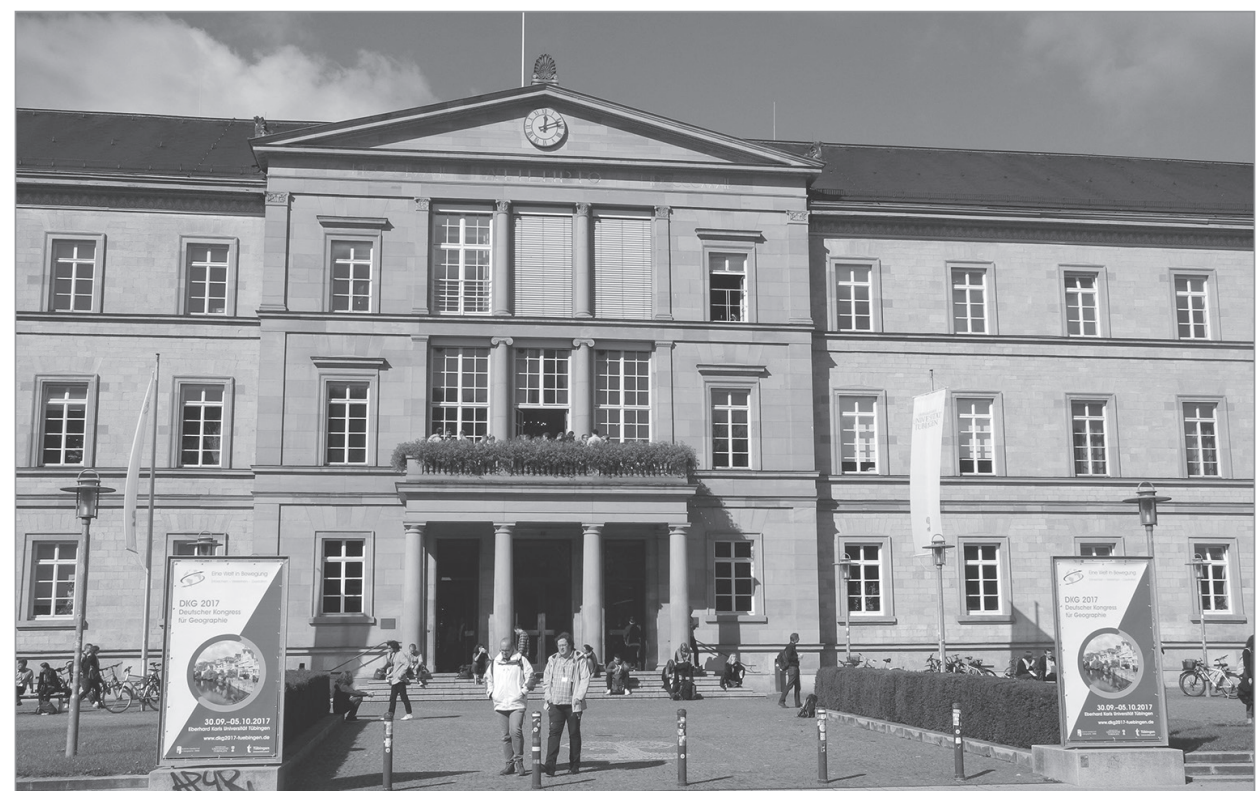

Foto: $\quad$ P.A. Rumpolt 2017

Abb. 2: Die Neue Aula der Eberhard-Karls-Universität Tübingen während des DKG 2017 


\section{Tagungsmotto und Programmgestaltung}

Der Kongress stand unter dem Motto „Eine Welt in Bewegung“ mit dem Untertitel „Erforschen - Verstehen - Gestalten“. Insgesamt 155 Fachsitzungen waren folgenden sieben Leitthemen zugeordnet (vgl. Deutsche Gesellschaft für Geographie \& Eberhard Karls Universität Tübingen 2017, S. 45ff.):

1. Migration und demographische Herausforderungen

2. Planet Stadt

3. Umwelt in Bewegung

4. Ökonomischer und sozialer Wandel

5. Natur und Gesellschaft

6. Methoden und Konzepte der Geographie

7. Geographische Bildung zukunftsfähig gestalten

Neben den diesen sieben Leitthemen entsprechenden Fachsitzungen war das Programm durch eine Reihe von altbewährten Formaten gekennzeichnet: So gab es ein umfassendes Exkursionsangebot zu Zielen in der engeren (Abb. 3) und weiteren Umgebung, Poster-Präsentationen (Abb. 4) sowie mehrere Keynote-Vorträge. Von den in das ,normale' Programm integrierten, zeitlich nicht ,alleingestellten' Keynote-Vorträgen waren sieben den Leitthemen zugeordnet, zwei weitere im Feld der Angewandten Geographie oder der Schulgeographie verortet.

Wie bereits zwei Jahre zuvor in Berlin wurden auch in Tübingen - ganz im Sinne der Ausrichtung des DKG auf die drei Bereiche Wissenschaft, Schule und Praxis - sowohl ein „Tag der Angewandten Geographie“ vom Deutschen Verband für Angewandte Geographie (DVAG) als auch

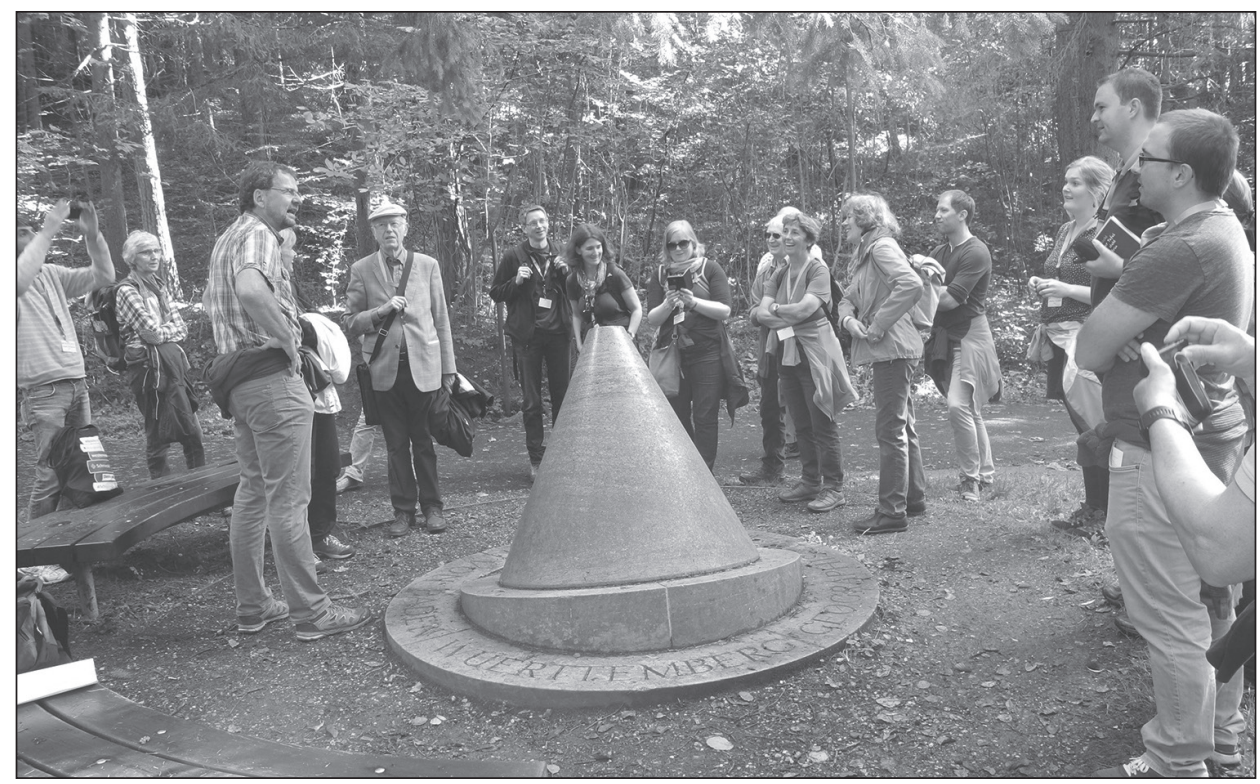

Foto: $\quad$ P.A. Rumpolt 2017

Abb. 3: Wanderexkursion „Naturraum und Kulturlandschaft von Tübingen“ unter der Leitung von Joachim EBERLE beim geographischen Mittelpunkt von Baden-Württemberg 


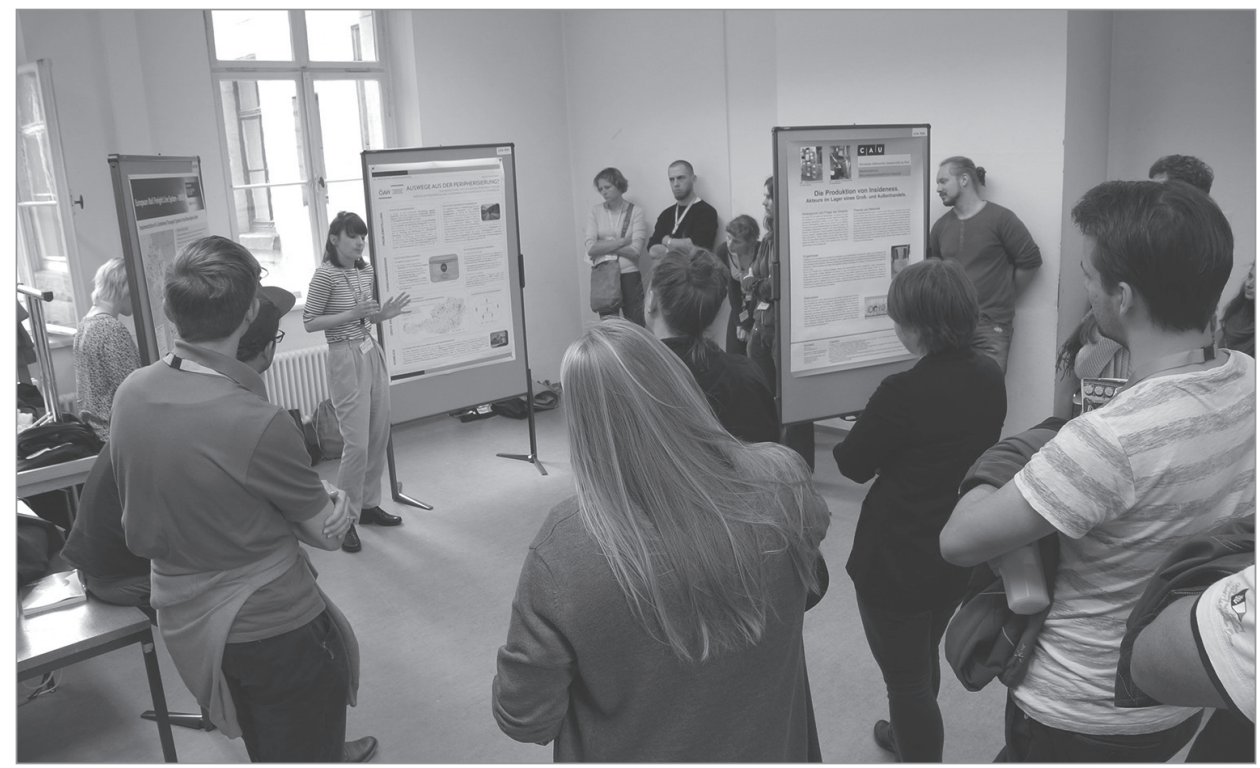

Foto:

P.A. Rumpolt 2017

\section{Abb. 4: Poster-Präsentation}

ein „Tag der Schulgeographie“ vom Verband Deutscher Schulgeographen (VDSG) abgehalten. An diesen beiden Tagen wurden spezielle Veranstaltungen für Geographen, die in der außeruniversitären Praxis oder in der Schule tätig sind, angeboten. Ebenso fortgeführt wurden der ,Junge Kongress für Geographie“ sowie die „Journal Lectures“ - insgesamt acht, welche sich mit den Fachsitzungen und den meisten Keynote-Vorträgen zeitlich nicht überschnitten.

Als Programmbestandteile neu aufgenommen wurden 2017 „Fachforen“ sowie offen gestaltete „World Cafés“, die den Leitthemen des Kongresses zugeordnet waren. Die drei Fachforen waren als öffentlich zugängliche Podiumsdiskussionen organisiert und hatten das Ziel, gesellschaftlich relevante Phänomene und Forschungsthemen der Geographie mit Akteuren von außerhalb der Wissenschaft sowie mit der interessierten Öffentlichkeit zu diskutieren. Das humangeographische Fachforum stand dabei unter dem Titel „Migration und Flucht“, das physiogeographische Fachforum widmete sich dem Themenkomplex „Naturrisiken und Umweltgefahren“, und im fachdidaktischen Fachforum bildete das Statement „Keine Bildung ohne Geographie!?“ den Gegenstand der Diskussion.

Das Programm des DKG 2017 wurde durch eine Reihe von Sonderveranstaltungen ergänzt, welche allgemeinere Fragen zur gegenwärtigen Wissenschafts- und Forschungspraxis diskutierten. So wurde in einer vom wissenschaftlichen Beirat des Verbandes der Geographen an Deutschen Hochschulen (VGDH) organisierten, gut besuchten Veranstaltung das interessante und wichtige Thema der Begutachtungskultur und Gutachtertätigkeit kritisch beleuchtet (Abb. 5), und zwar sowohl bezogen auf Zeitschriftenartikel als auch auf Forschungsvorhaben und Berufungsverfahren. Unter dem Titel „Interdisziplinäre Praxis einer Natur/Gesellschafts-Forschung“ wiederum diskutierten Human- und Physiogeographen in einer weiteren Sonderveranstaltung gemeinsam Chancen und Herausforderungen ,integrativer' Forschungsprojekte. Außerdem gab es eine vergleichsweise kleine, aber feine (und diesmal in einem Zelt untergebrachte) Fach- und Verlagsausstellung (Abb. 6); der Westermann-Verlag oder die Westermann-Gruppe war diesmal nicht vertreten. 


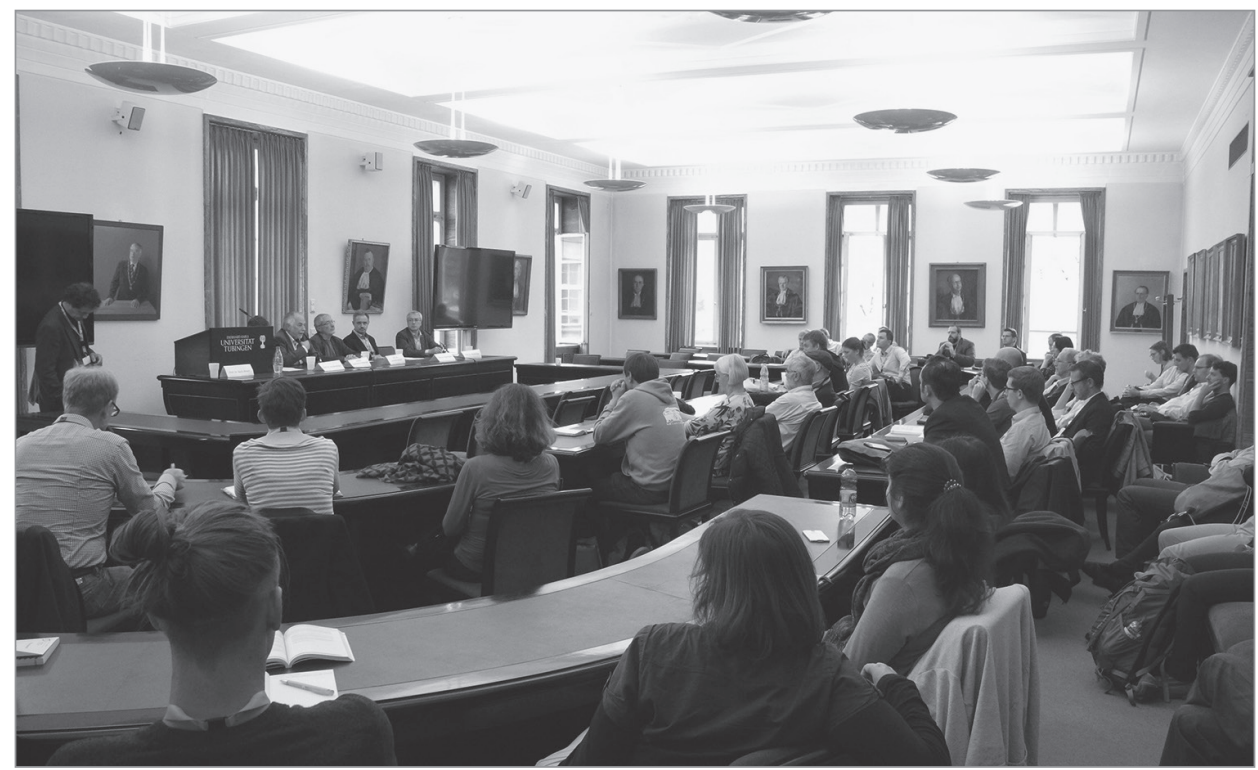

Foto: P.A. Rumpolt 2017

Abb. 5: Podiumsdiskussion zur Begutachtungskultur in der Geographie mit Hans-Heinrich BLotevOGEL als einem der Diskutanten am Podium

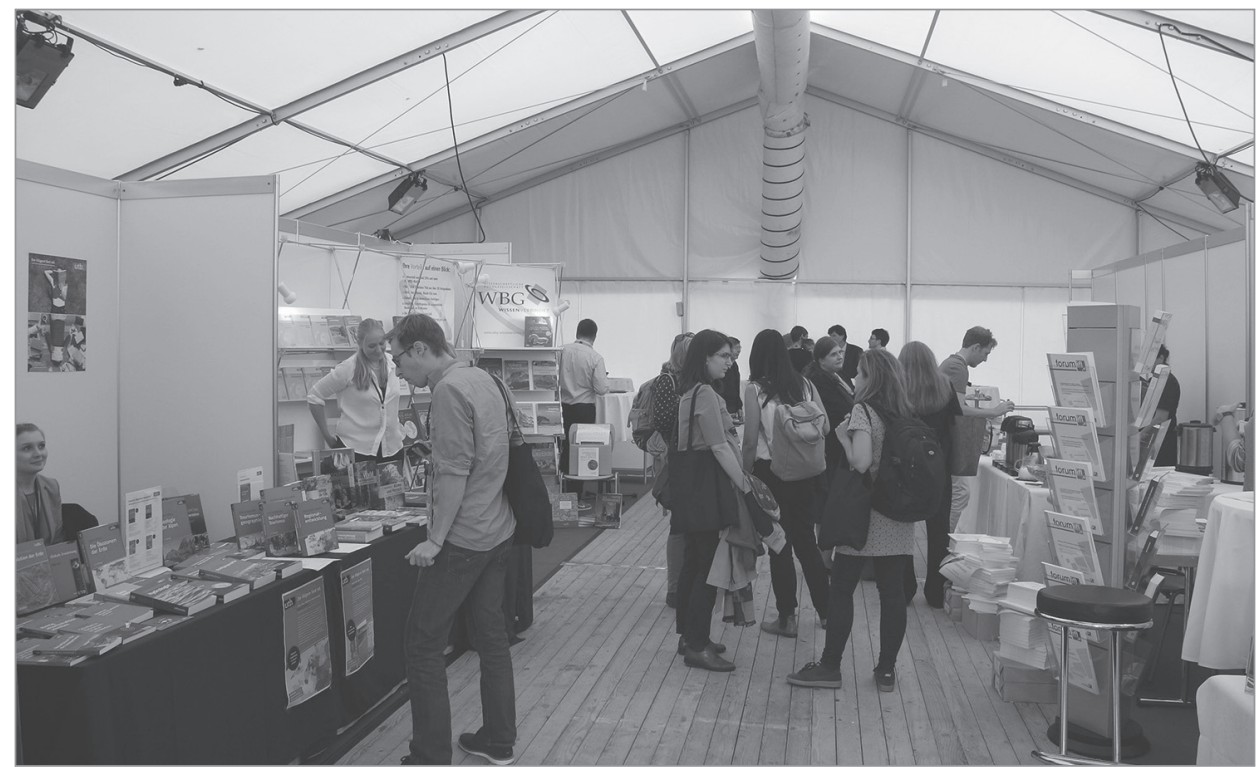

Foto: $\quad$ P.A. RumpoLt 2017

Abb. 6: Die in einem Zelt untergebrachte Fach- und Verlagsausstellung 
Das Rahmenprogramm beinhaltete die Festveranstaltung am Abend des 30. September 2017 im Festsaal der Neuen Aula, in welcher der Philosoph Otfried HöFfE die Festrede zur Frage „Was hält Europa zusammen?“ hielt und in deren Rahmen wissenschaftliche Preise verliehen wurden. Der Tübinger Oberbürgermeister Boris PALMER freute sich in seinem Grußwort scherzhaft über die Erwähnung Stuttgarts als Teil der Umgebung Tübingens im Exkursionsführer, und Werner GAMERITH, Präsident der Deutschen Gesellschaft für Geographie (DGfG), plädierte in dem seinen dafür, Forschungsergebnisse der Geographie in die Öffentlichkeit zu tragen und sich dabei auch als Geographen zu deklarieren und nicht z.B. als Stadt- oder Klimaforscher ,umzuetikettieren“. Zum Rahmenprogramm zählte auch ein „Get-Together“, das am 2. Oktober 2017 abends in den eher schmucklosen Räumlichkeiten einer Mensa stattfand und bei dem man sich in lockerer Atmosphäre zum gemeinsamen Abendessen traf.

\section{Fachpolitische Reflexionen}

Aus fachpolitischer Sicht verdienen besonders drei Ereignisse oder Aspekte des DKG 2017 eine (kritische) Reflexion:

- das Abhalten eines „Parallel-Kongresses“;

- die Diskussionen um das humangeographische Fachforum „Migration und Flucht“;

- die inhaltliche Einengung des DKG auf (zu) wenige Themenbereiche.

Bereits im Vorfeld des DKG 2017 war von einigen Seiten, vor allem von Vertretern einer Kritischen Geographie und aus dem Bereich der Neuen Kulturgeographie, Kritik an der Auswahl der Fachsitzungen durch den Ortsausschuss geäußert worden. Dies führte zu einem vom 29. September bis 1. Oktober vom DGfG-Arbeitskreis „Kritische Geographie“ parallel durchgeführten „Kongress der Kritischen Geographie“ (KKG). Den Organisatoren zufolge beteiligten sich ca. 140 Personen an insgesamt 16 Veranstaltungen (vgl. LEAL Soto et al. o.J.).

Das Abhalten einer ,Alternativ-Tagung' zur gleichen Zeit und am gleichen Ort stellt natürlich ein fachpolitisches Statement dar und kann im Sinne einer offenen und pluralistischen Disziplin auch begrüßt werden. Kritisch angemerkt sei jedoch, dass die organisatorische und inhaltliche Zersplitterung des DKG vermieden werden sollte - im Hinblick auf die Außenwahrnehmung der Disziplin, aber auch auf das Ziel des Aufrechterhaltens einer paradigmenübergreifenden Diskussionsbasis durch gemeinsam getragene Kongresse.

Das Faktum der multiparadigmatischen Struktur des Faches ist hinlänglich bekannt. Gerade wichtige Fachkongresse sollten diese Struktur in einem gemeinsamen organisatorischen und inhaltlichen Rahmen abbilden. Künftigen Kongressen wäre es daher anzuraten, einerseits über die Ortsausschüsse möglichst alle relevanten Ansätze und Forschungsrichtungen der Geographie einzubinden und andererseits von den Arbeitskreisen den Willen zur Diskussion im Rahmen einer „,integrativen" Tagung, die inhaltlich offen für Neues und Anderes ist, einzufordern.

Das in Tübingen neu eingeführte Format der „Fachforen“, die als öffentlich zugängliche Veranstaltungen Wissenschaft mit Politik und Gesellschaft verbinden sollten, stand im Bereich der Humangeographie unter dem Titel „Migration und Flucht“ (Abb. 7). Dieser Themenkomplex wurde im Rahmen einer Podiumsdiskussion erörtert, wobei das Podium aus mehreren Fachvertretern der Geographie und dem Tübinger Oberbürgermeister Boris PALmER (Bündnis 90/Die Grünen) zusammengesetzt war. Dessen Einladung und Teilnahme war in einem offenen Brief an die Veranstalter scharf kritisiert worden, und diese Kritik hatte bereits vor dem Kongress zu Berichten in lokalen Medien geführt. In diesem offenen Brief, der von etlichen Geographen an deutschsprachigen Hochschulen 
unterzeichnet war, wurden die Positionen von Boris PALMER zur deutschen Flüchtlingspolitik scharf kritisiert und als „ausländerfeindlich“ und „,rassistisch“ qualifiziert. ${ }^{4)}$ Im Laufe der Veranstaltung kam es zu sehr kontroversiell und emotional geführten Debatten zwischen Boris PALMER und Teilen des Publikums, sodass eine sachliche Diskussion kaum möglich war und andere Podiumsdiskutanten entsprechend wenig zu Wort kamen.

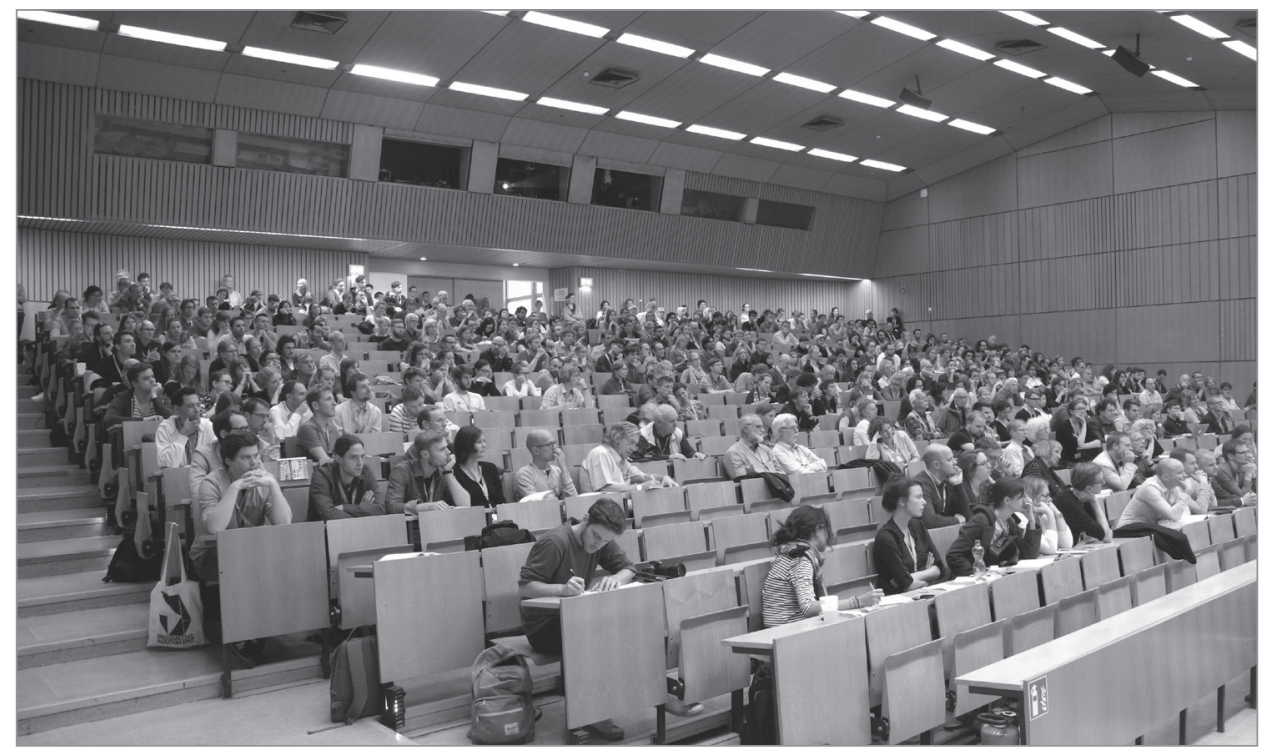

Foto: $\quad$ P.A. Rumpolt 2017

Abb. 7: Großes Interesse an der öffentlich zugänglichen Podiumsdiskussion zu Migration und Flucht

An diesem Beispiel wird zunächst deutlich, dass ein Austausch zwischen Wissenschaft, Politik und Gesellschaft wichtig ist und dass die Geographie dabei eine wichtige Rolle spielen kann. Es kann jedoch - wie man sieht - auch passieren, dass sich die Diskussion gänzlich in die politische Sphäre verlagert und wissenschaftliche Evidenz in Form von empirischen Fakten und theoretisch-konzeptionell fundierten Argumenten völlig in den Hintergrund gerät. So klassifizierte Boris PAlmer die sehr kritischen Kommentare aus dem Publikum einmal als Aussagen aus dem „Elfenbeinturm“. Umgekehrt verweigerten Diskutanten aus dem Publikum eine Auseinandersetzung mit den konkreten Herausforderungen der Lokalpolitik durch die Integration von Flüchtlingen, wie sie vom Oberbürgermeister angesprochen wurden, und lenkten die Debatte auf die abstrakte Ebene globaler Zusammenhänge und Machtstrukturen.

Eine Verständigung von Wissenschaft und Politik fand somit nicht statt, und es stellt sich die Frage, ob Wissenschaft nicht besser auf politische Entscheidungen und Entscheidungsträger einwirken könnte, wenn sie auf der Basis valider Erkenntnisse einen aufgeklärten Blick auf Phänomene unserer Zeit in verständlicher Form verdeutlicht und diesen gegenüber der Politik und der Gesellschaft aktiv, aber immer auch sachlich vertritt.

4) Der offene Brief kann auf der Webseite des AK Kritische Geographie nachgelesen werden: http://kritischegeographie.de/offener-brief-migrationundflucht/ (letzter Zugriff 21.12.2017) 
Der inhaltlichen Breite unserer Disziplin könnte in Zukunft auch wieder durch die Auswahl der Fachsitzungen besser Rechnung getragen werden. Eine bereits bei den vorangegangenen beiden Geographentagen in Passau und Berlin sichtbare Unterrepräsentanz der Physischen Geographie bei Fachsitzungen (vgl. Rumpolt 2013a, S. 326; Rumpolt \& Seebacher 2015a, S. 367) war auch in Tübingen zu beobachten. Zu starke Fokussierungen auf einen Teilbereich der Geographie im Allgemeinen sowie auf wenige Themenkomplexe im Speziellen - in Tübingen fielen z.B. die Themenfelder Migration und urbane Räume auf - sollten möglichst vermieden werden, auch dann, wenn es sich dabei um (auch gesellschaftlich und politisch) besonders aktuelle und relevante Fragen handelt.

\section{Reflexionen zur Organisation}

Zunächst seien Gestaltung, Struktur und Übersichtlichkeit des Programmheftes ausdrücklich gelobt. Eine entsprechende Anmerkung der Autoren zum Programmheft des vorangegangenen Kongresses in Berlin dürfte Gehör gefunden haben (siehe Rumpolt \& Seebacher 2015a, S. 367f.). Die Strukturierung der Fachsitzungen im Programmheft nach Leitthemen erwies sich in Kombination mit der im Anschluss an die Grußworte abgedruckten Programmübersicht als sehr praktisch. Speziell diese graphisch gut gelöste Programmübersicht, welche sämtliche Veranstaltungsformate sowie bei jeder Veranstaltung auch einen Seitenverweis beinhaltet, erleichterte die rasche Orientierung in dem doch komplexen Gesamtprogramm wesentlich. Die Orientierung während der Tagung und damit das Wechseln zwischen verschiedenen Sitzungen wurden durch Übersichtslisten über die in einem bestimmten Raum stattfindenden Veranstaltungen im jeweiligen Eingangsbereich erleichtert.

Ob es günstig ist, die ersten beiden der insgesamt vier ,Hauptkongresstage' an ein Wochenende zu legen, mag unterschiedlich beurteilt und empfunden werden. Dass aber Sitzungen der DGfG-Arbeitskreise zwar in Verbindung mit dem Kongressprogramm angekündigt werden, jedoch allem Anschein nach doch nicht integraler Bestandteil des DKG sind, ist sehr zu hinterfragen. Warum gelten die Arbeitskreissitzungen als am Tag vor (29.09.) dem offiziellen Kongress (30.09.-05.10.) stattfindend, während am letzten offiziellen Kongresstag (05.10.) keine Veranstaltungen mehr am Programm standen? Wieso hat das Tagungsbüro am Tag der Arbeitskreissitzungen nur zeitgleich mit diesen und nicht vielleicht auch schon davor geöffnet? Warum finden Vorsitzende von Arbeitskreisen ihren Veranstaltungsraum in einem unvorbereiteten Zustand vor (keine geordnete Aufstellung von Tischen und Sesseln) und warum müssen sie, um Präsentationen zu ermöglichen, ihren eigenen Laptop bereitstellen? Wieso ist zu diesen Sitzungen niemand dauerhaft abgestellt, der bei technischen Problemen hilft? Eine stärkere Integration der Sitzungen von DGfG-Arbeitskreisen in das Programm eines Geographentages könnte jedenfalls einer Überlegung wert sein.

Einen weiteren Problemfall bildeten die Poster-Präsentationen. Wie dem gedruckten Programmheft zu entnehmen ist, war jedes Poster innerhalb einer Stunde neben etlichen anderen zur mündlichen Vorstellung durch den Autor (inkl. anschließender Möglichkeit zur Diskussion) vorgesehen. Dass die ,Moderation“ dieser Stunde und das Einhalten des zeitlichen Ablaufs einem zufällig anwesenden Kongressteilnehmer anvertraut wurden - nur eine kurze organisatorische Einführung war zuvor durch ein Mitglied des „DKG-Teams“ vorgenommen worden -, war eine spannende, aber in der Praxis nicht immer funktionierende Idee. Eventuell hätten die Poster-Präsentationen zum Leitthema „Methoden und Konzepte der Geographie“ aufgeteilt auf zwei nach Physischer Geographie und Humangeographie differenzierten Terminen erfolgen können.

Als wirklich ärgerlich erwies sich aber die Tatsache, dass Poster wesentlich früher als angekündigt (teilweise schon am zweiten der vier Hauptkongresstage) und ohne Vorinformation abgenommen wurden - dies, obwohl sich die jeweils angegebene Stunde laut Programmheft eben nur auf die mündliche Vorstellung bezöge (vgl. Deutsche Gesellschaft für Geographie \& Eberhard 
Karls Universität TüBIngen 2017, S. 235) - und den Autoren vor Beginn der Tagung durch die Kongressorganisation angekündigt worden war, dass die Poster über einen längeren Zeitraum ausgestellt bleiben könnten (z.B. „Dauer der Posterausstellung in diesem Raum: 30.09.-03.10.“).

Abschließend sei hier noch kurz auf die Verköstigung am Tagungsgelände eingegangen: Die vor zwei Jahren seitens der Autoren zur Nachahmung empfohlene Möglichkeit, in den Pausen zwischen den Sitzungen kostenlos Fruchtsäfte, Kaffee, Kekse und Ähnliches konsumieren zu können (vgl. Rumpolt \& SeEbacher 2015a, S. 367), bestand erfreulicherweise auch während des DKG 2017 in Tübingen. Dabei wurde sogar Apfelsaft aus der Region angeboten. Ob es allerdings sinnvoll ist, diese Möglichkeit auf bestimmte Zeiträume einzuschränken, in denen dann die gleichzeitige Nachfrage besonders Vieler zu Wartezeiten und Angebotsengpässen führt, sei dahingestellt.

\section{Ausblick}

Im Rahmen der Festveranstaltung verkündete der Präsident der DGfG, Werner GAMERITH, dass bereits die Austragungsorte der nächsten vier (!) Deutschen Kongresse für Geographie (2019, 2021, 2023, 2025) feststünden bzw. weitgehend gesichert seien. Der nächste DKG wird 2019 in Kiel (Schleswig-Holstein) stattfinden. Genau 50 Jahre nach dem „Kieler Geographentag“"von 1969, welcher in der Geschichte der deutschsprachigen Geographie einen wichtigen Wendepunkt markiert, kehrt man also mit dem Fachkongress dorthin zurück. Anzunehmen ist, dass im Rahmen der Tagung in Kiel ein besonderer Schwerpunkt auf eine Auseinandersetzung mit der Geschichte der Geographie - speziell auch in wissenschaftstheoretischer und paradigmatischer Hinsicht - gelegt wird. Bereits in den vergangenen Jahren wurde die „Kieler Wende“ inklusive ihrer Hintergründe, Ursachen und Folgen in verschiedenen Veröffentlichungen neu aufgearbeitet (siehe Korf 2014; WeICHHART 2016). Im Jahr 2021 wird der Deutsche Kongress für Geographie dann in Frankfurt am Main (Hessen) abgehalten. Im Jahr 2023 wird man sich voraussichtlich im Ruhrgebiet (Nordrhein-Westfalen) einfinden; als Austragungsort(e) wurde(n) Bochum, eventuell gemeinsam mit Duisburg-Essen, kolportiert. Und für das Jahr 2025 könnte es hinsichtlich der Örtlichkeit eine Überraschung sowie eine Premiere im deutschen Sprachraum geben.

\section{Literaturverzeichnis}

Deutsche Gesellschaft für Geographie (DGfG), Eberhard Karls Universität Tübingen (Hrsg.) (2017), Eine Welt in Bewegung. Erforschen - Verstehen - Gestalten. DKG 2017. Deutscher Kongress für Geographie. Programm, Tübingen.

Gamerith W. (2017), Grußwort der Deutschen Gesellschaft für Geographie (DGfG). In: Deutsche Gesellschaft für Geographie (DGfG), Eberhard Karls Universität Tübingen (Hrsg.), Eine Welt in Bewegung. Erforschen - Verstehen - Gestalten. DKG 2017. Deutscher Kongress für Geographie. Programm, S. 11. Tübingen.

Kinder S. (2017), Grußwort des Ortsausschusses Tübingen. In: Deutsche Gesellschaft für Geographie (DGfG), Eberhard Karls Universität Tübingen (Hrsg.), Eine Welt in Bewegung. Erforschen - Verstehen - Gestalten. DKG 2017. Deutscher Kongress für Geographie. Programm, S. 12. Tübingen.

Korf B. (2014), Kiel 1969 - ein Mythos? In: Geographica Helvetica, 69, 4, S. 291-292. - https:// doi.org/10.5194/gh-69-291-2014.

Leal Soto C., Hoinle B., Petzold T., Schweizer P. (o.J.), Bericht zum Kongress der Kritischen Geographie, Tübingen vom 29.09. - 01.10.2017. Nicht redigierte Fassung. - http://kriti- 
sche-geographie.de/wp-content/uploads/2017/12/Bericht-fuer-Rundbrief_fürHomepage. pdf (letzter Zugriff 21.12.2017).

Musil R. (2009), Der Deutsche Geographentag in Wien 2009 - eine wissenschaftliche Großveranstaltung. In: Mitteilungen der Österreichischen Geographischen Gesellschaft, 151, S. 315-322.

Nissel H., Embleton-Hamann C. (2012), Down to Earth - IGC Cologne 2012. 32. Weltkongress der Geographie in Köln, August 2012. In: Mitteilungen der Österreichischen Geographischen Gesellschaft, 154, S. 341-346. - https://doi.org/10.1553/moegg154s317

Rumpolt P.A. (2013a), Der 58. Deutsche Geographentag 2013 in Passau - ein großer Kongress in einer kleinen Stadt. In: Mitteilungen der Österreichischen Geographischen Gesellschaft, 155, S. 321-326. - https://doi.org/10.1553/moegg155s303

Rumpolt P.A. (2013b), Geographentag 2013 in Passau - ein Rückblick. In: Geographie aktuell. Informationen der Österreichischen Geographischen Gesellschaft, 5, 4, S. 3. - http://www. geoaustria.ac.at/items/uploads/files/ga2013_4_online.pdf (letzter Zugriff 21.12.2017).

Rumpolt P.A., Seebacher M.M. (2015a), Der Deutsche Kongress für Geographie 2015 in Berlin - ein kritischer Rückblick. In: Mitteilungen der Österreichischen Geographischen Gesellschaft, 157, S. 363-369. - https://doi.org/10.1553/moegg157s363

Rumpolt P.A., SeEbacher M.M. (2015b), Deutscher Kongress für Geographie 2015 in Berlin - ein Rückblick. In: Geographie aktuell. Informationen der Österreichischen Geographischen Gesellschaft, 7, 4, S. 5. - http://www.geoaustria.ac.at/items/uploads/files/geoaktuell_26_2015_ online.pdf (letzter Zugriff 21.12.2017).

Rumpolt P.A., Seebacher M.M. (2017), Deutscher Kongress für Geographie 2017 in Tübingen ein Rückblick. In: Geographie aktuell. Informationen der Österreichischen Geographischen Gesellschaft 9, 4, S. 3. - http://www.geoaustria.ac.at/items/uploads/files/geo_aktuell34_online.pdf (letzter Zugriff 21.12.2017).

WeichHart P. (2016), Der Kieler Geographentag 1969 - Modernisierungsschub, Mythos, Paradigmenwandel oder vergessene Geschichte? In: Geographica Helvetica, 71, 1, S. 7-13. https://doi.org/10.5194/gh-71-7-2016 